\title{
Diagnosis of Invasive Non-functional Pituitary Adenomas by Serum Extracellular Vesicles
}

\author{
Huayi Wang ${ }^{\S, /, \Phi,+}$, Kelin Chen ${ }^{\ddagger,+}$, Zhijun Yang ${ }^{\ddagger,+}$, Wenzhe Li ${ }^{\S, \Phi, ~}$, , Chen Wang ${ }^{\S, \Phi}$, Guojun

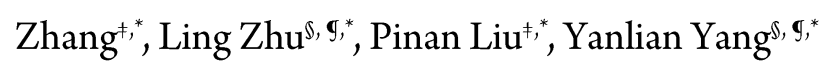 \\ ${ }^{\S}$ CAS Key Laboratory of Standardization and Measurement for Nanotechnology, CAS Key Laboratory of \\ Biological Effects of Nanomaterials and Nanosafety, CAS Center for Excellence in Nanoscience, National \\ Center for Nanoscience and Technology, Beijing 100190, China \\ ${ }^{\ddagger}$ Beijing TianTan Hospital, Capital Medical University, Beijing, 100070, China \\ "Department of Chemistry, Tsinghua University, Beijing, 100084, China \\ "University of Chinese Academy of Sciences, 19 A Yuquan Rd, Shijingshan District, Beijing, 100049, China \\ ${ }^{\dagger}$ Academy for Advanced Interdisciplinary Studies, State Key Laboratory Of Natural And Biomimetic Drugs, \\ School of Pharmaceutical Sciences, Peking University, 100871, China \\ ${ }^{\dagger}$ These authors contributed equally to this work.
}




\section{Supporting Information}

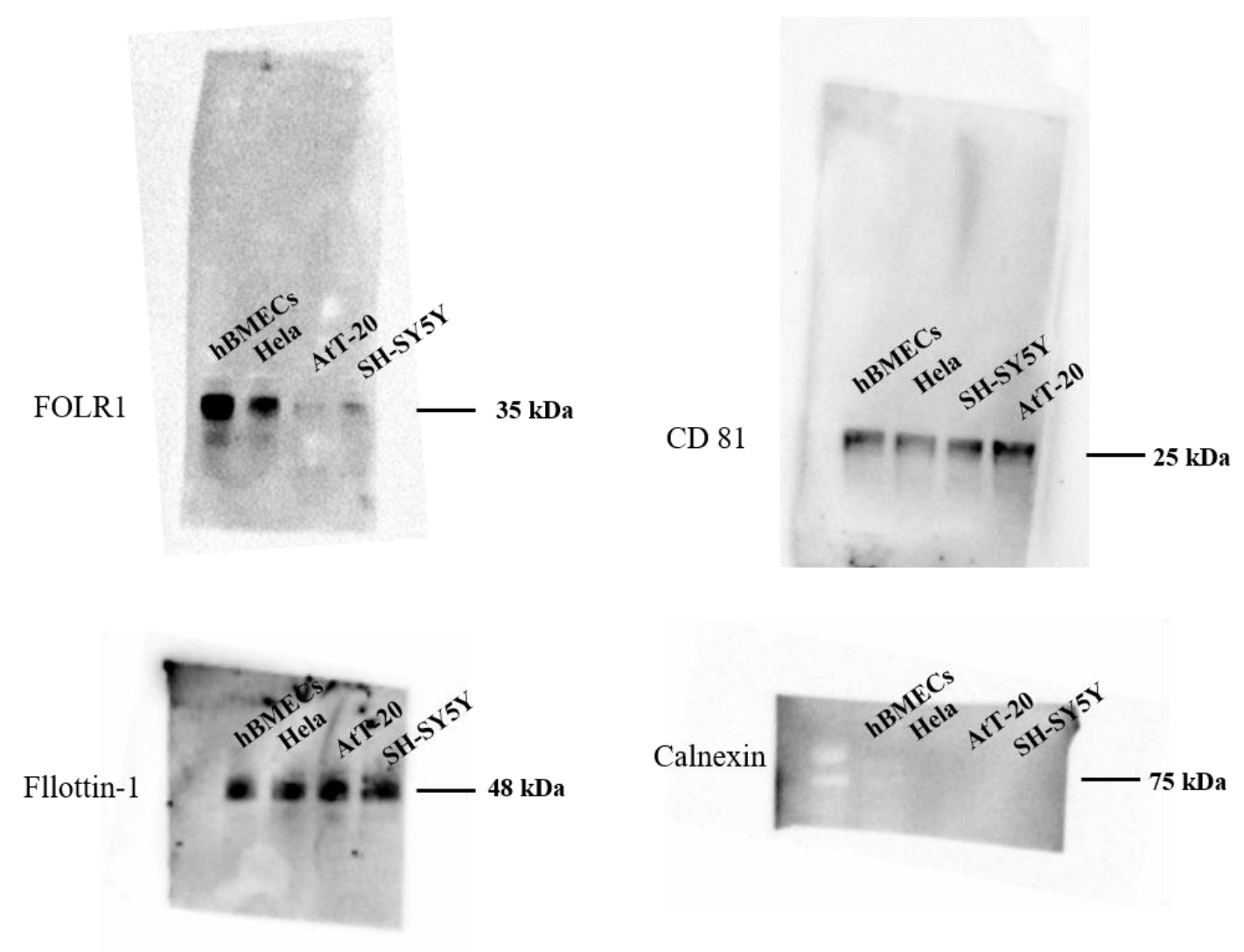

Figure S-1. Uncropped full-length blots of the expression of FOLR1, Flotillin-1, CD81,

Calnexin on EVs from hBMECs and AtT-20 cell lines. 


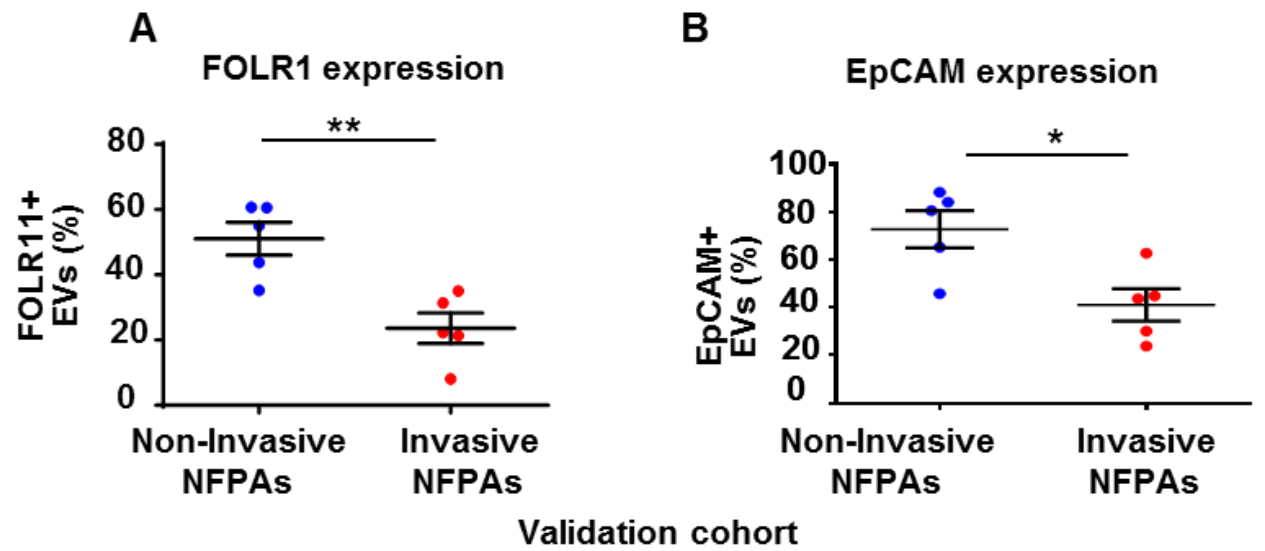

Figure S-2. Validation cohort of invasive and non-invasive patients using serum EVs. 


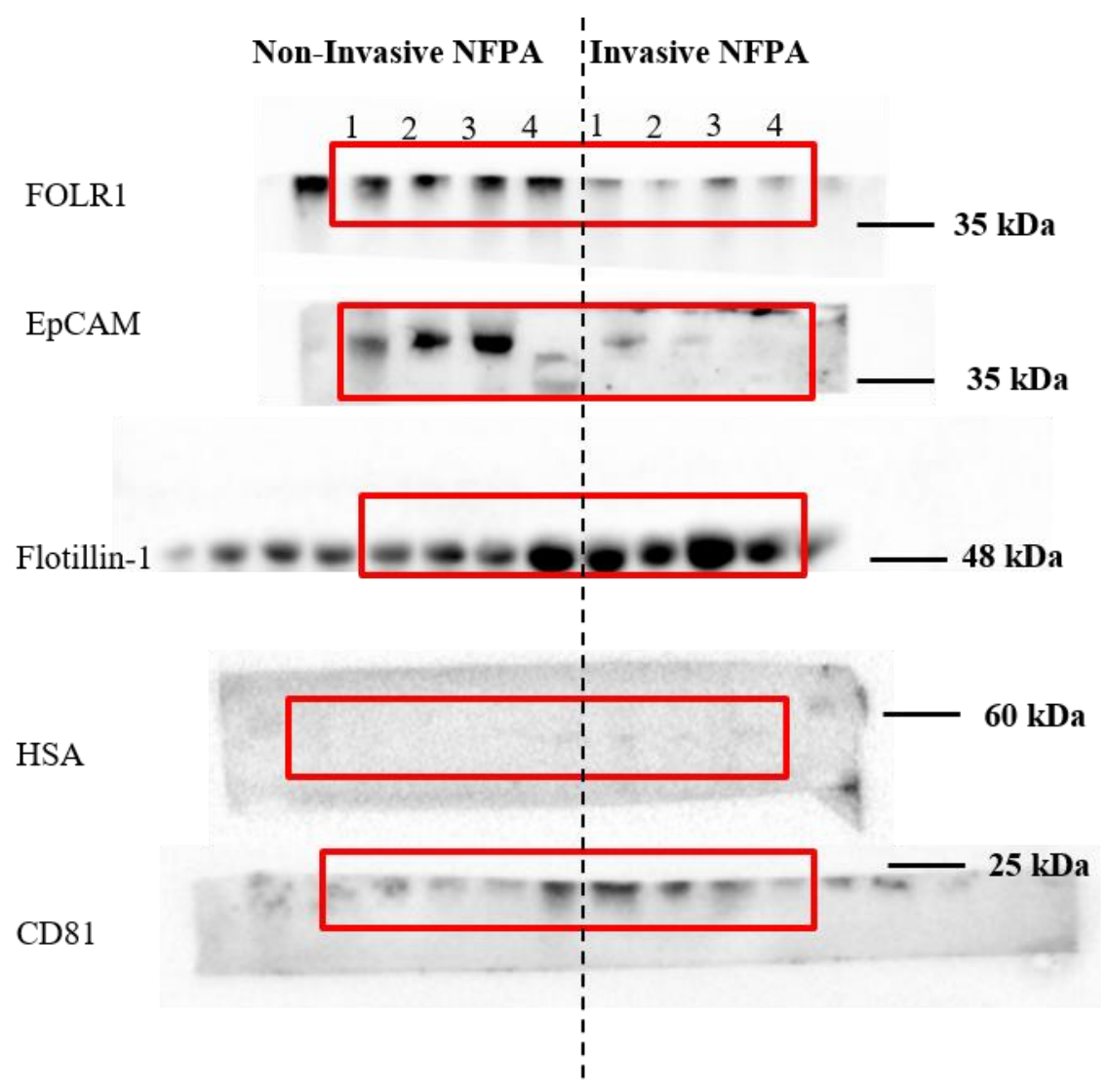

Figure S-3. Uncropped full-length blots of the expression of FOLR1, EpCAM, Flotillin1, HSA, CD81 on serum EVs from patients. 
A

Non Invasive NFPAs
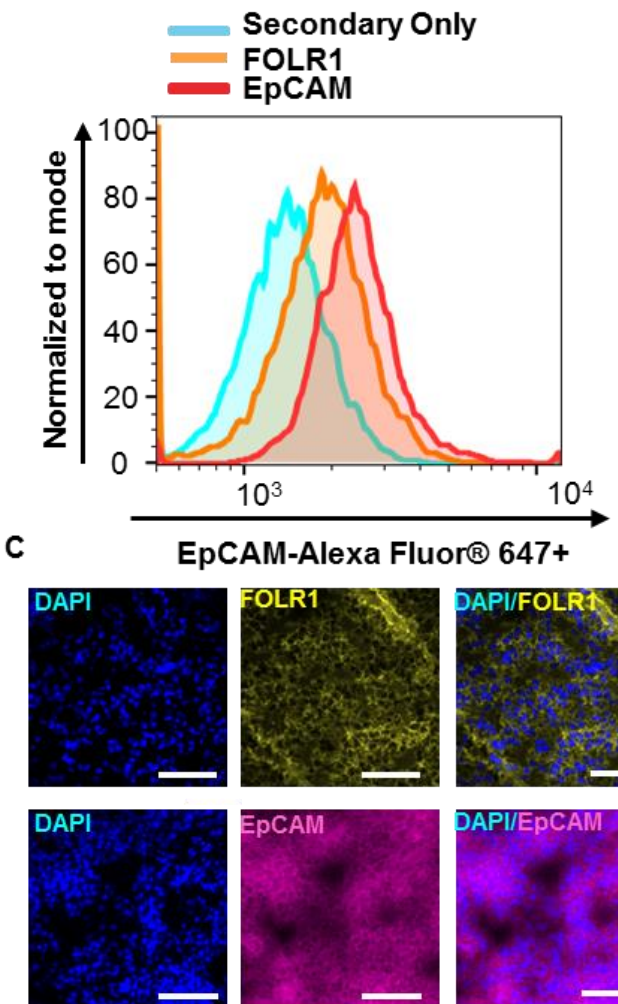

DAPI
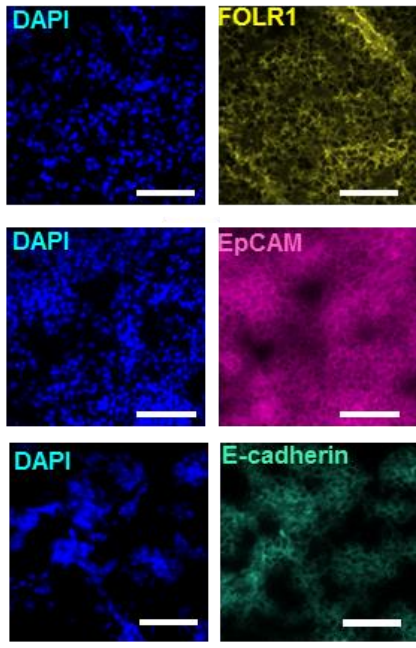

DAPI

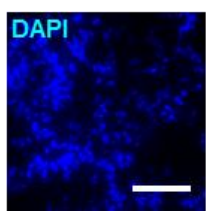

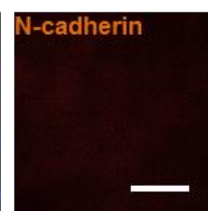

B
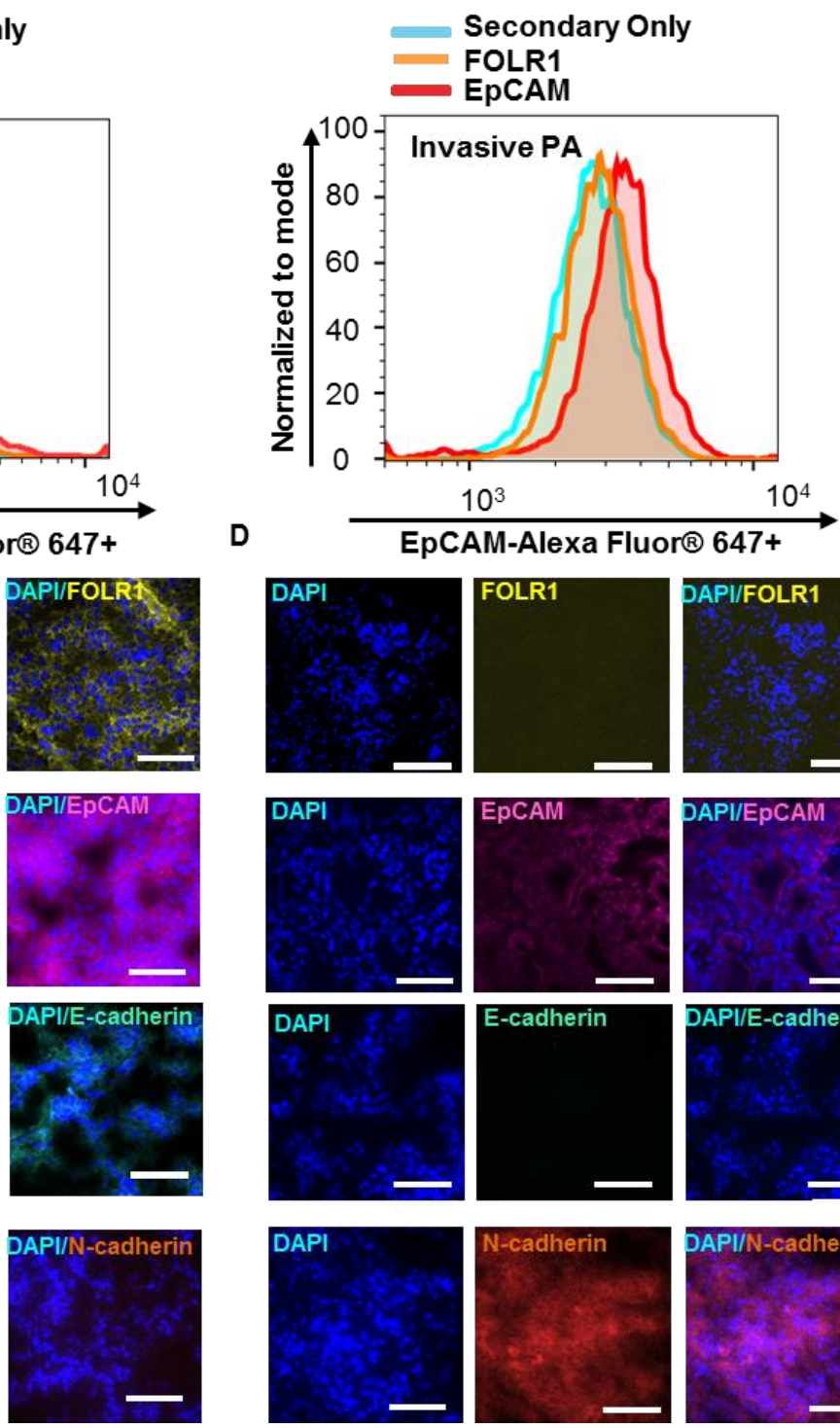
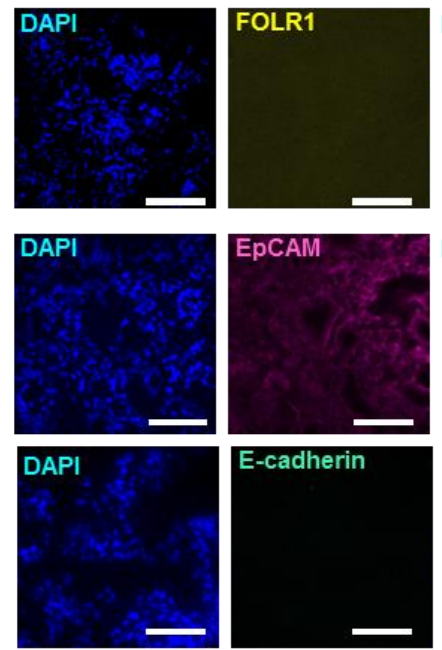

E-cadherin
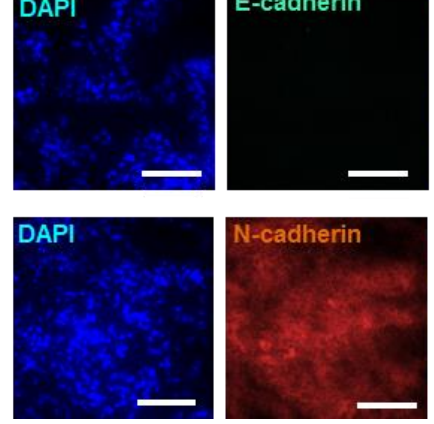

Figure S-4. Epithelial-mesenchymal transition (EMT) tendency in the tumor tissues from the

invasive NFPAs patients. (A) and (B) Representative flow cytometry analysis of the expression of EpCAM and FOLR1 in serum EVs from one non-invasive (A) and one invasive NFPAs patient (B). (C) and (D) Immunofluorescent staining showing the expression of FOLR1, EpCAM, E-cadherin and N-cadherin in the tumor tissue from the corresponding non-invasive $(\mathrm{C})$ and invasive NFPAs patients (D). 
A

Non Invasive NFPAs
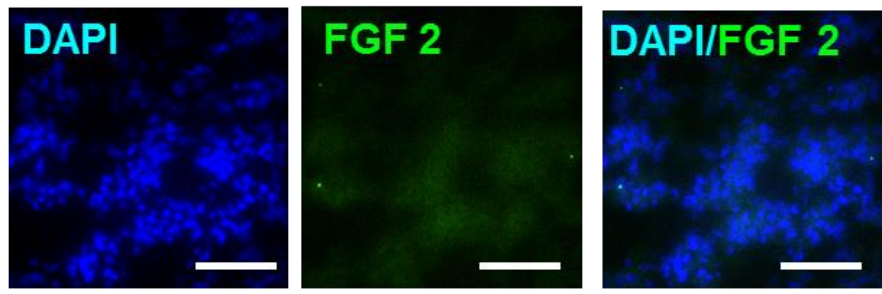

Invasive NFPAs
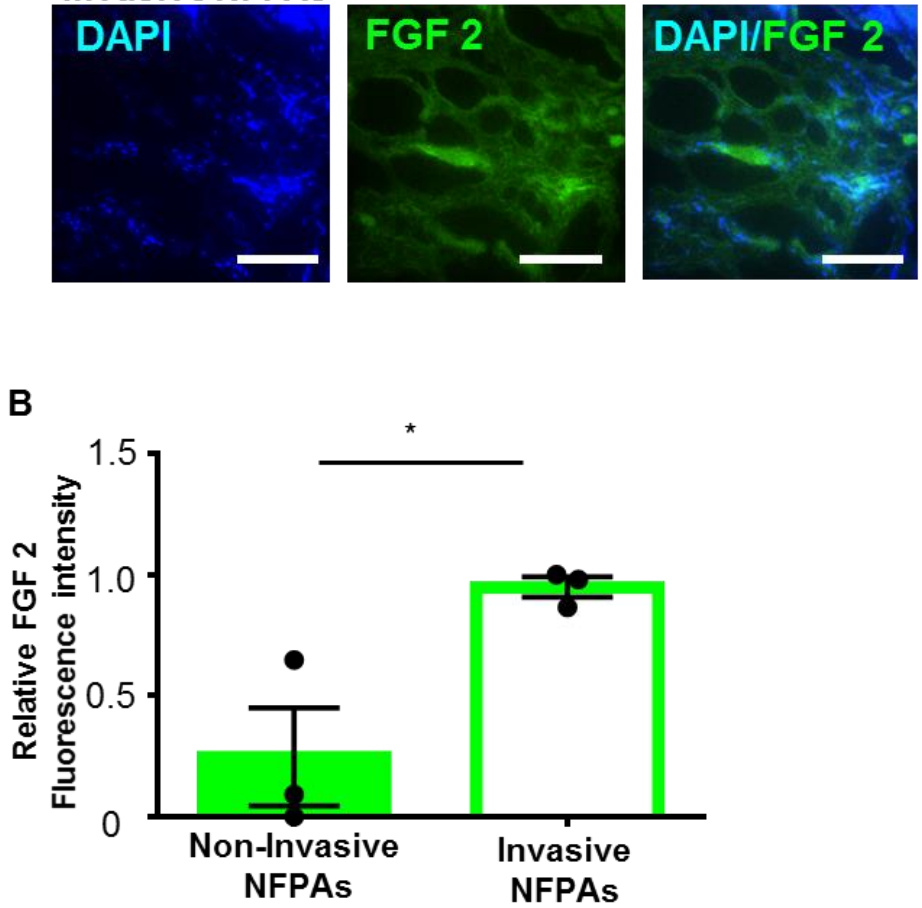

Figure S-5. (A) Fluorescent imaging of the expression of FGF 2 in the tumor tissue of one onoinvasive and one invasive NFPAs patient. (B) Normalized expression level of FGF 2 in tumor tissues from non-invasive $(\mathrm{n}=3)$ and invasive $(\mathrm{n}=3)$ NFPAs patients. (unpaired Student's t-test , *, $\mathrm{P}<0.05)$. 
Table-1. Receiver operating characteristic (ROC) analysis of discriminative efficiency of FOLR1, EpCAM and EVs protein concentration.

\begin{tabular}{ccccc}
\hline & Area & Std. Error & $\begin{array}{c}95 \% \text { confidence } \\
\text { interval }\end{array}$ & P value \\
\hline FOLR1+ EVs & 0.94 & 0.05455 & 0.8331 to 1.047 & 0.0008871 \\
EpCAM+ EVs & 0.88 & 0.07761 & 0.7278 to 1.032 & 0.004089 \\
\hline $\begin{array}{c}\text { EVs protein } \\
\text { concentration }\end{array}$ & 0.50 & 0.1307 & 0.2438 to 0.7652 & 1.000 \\
\hline
\end{tabular}

